\title{
Images et représentations du cancer
}

\author{
P. Guex \\ (C) Springer-Verlag 2009
}

L'éditorial du rédacteur invité devrait, semble-t-il, apporter un commentaire à quatre articles originaux du monde francophone, dont deux issus du Maghreb, et deux de Suisse, l'un par un Romain d'origine comme il se doit dans notre pays dont $30 \%$ de la population est «étrangère » et l'autre par moi-même binational suisse-français. Cela devrait être un terrain d'observation idéal pour aborder le thème «Images et représentations du cancer » correspondant à des constructions sociales, culturelles, et individuelles probablement non identiques entre le Maroc, l'Algérie, la France et la Suisse.

Identifier et étudier la diversité des représentations du cancer c'est traverser en effet les normes médicales et administratives d'un côté et le vécu des malades et des soignants de l'autre, au carrefour de l'offre sociale.

Le magnifique article scientifique de Leila Hessissen et collègues sur «L'impact psychologique et social chez des adolescents traités pour cancer pendant l'enfance » ne laisse que peu transparaître de problématiques transculturelles tant ses adolescents ressemblent à s'y méprendre aux nôtres dans leurs réactions, leurs désirs, leur rapport au corps et leur souci de dépasser l'événement traumatique. Comme chez les Suisses, surtout en biomédecine, la référence au monde anglo-saxon est le standard, même si parfois cela se distancie de nos expériences quotidiennes.

Il en est tout autre pour le Dr Mustapha Maaoui qui grâce à son expérience nous entraîne dans un hallucinant voyage « de la colonisation à la régression : le point en Algérie ». Cette traversée historique met en valeur la notion anthropologique de système médical pluraliste à la fois colonial et « indigène », puis au sein même de la société algérienne, en fonction d'un certain contexte politique, à travers trois secteurs classiques : un secteur populaire ou vernaculaire, un secteur de spécialistes de la santé (professionnels biomédicaux et non biomédicaux de type occidental) et un secteur traditionnel caractérisé par des approches diversifiées émanant d'expériences culturelles et religieuses différentes.

P. Guex $(\square)$

Chef de département, Département de Psychiatrie

CHUV, Rue du Bugnon 44CH - 1011 Lausanne

e-mail : patrice.guex@chuv.ch
Cette évolution algérienne vers les chamanes et les médecines complémentaires rencontre les mêmes développements en Suisse même si nous ne nous trouvons pas au carrefour de plusieurs expressions de l'islam.

Daniele Stagno pour sa part, avec sa sensibilité de psychothérapeute psychanalyste, ouvre un nouveau champ culturel : celui de la distinction entre les représentations sociales dans le discours scientifique ou préventif et les représentations intimes, liées à l'imaginaire individuel. Ces dernières permettent de progresser dans l'intériorisation d'une expérience dans le face à face et l'intersubjectivité avec le soignant.

Cela me permet une transition de type culturel sur notre travail en médecine avec les populations migrantes dans notre pays. Quelle que soit leur forme, les thérapies et la prévention se rattachent à la communication médicale dans son acception la plus large. De ce fait, les expériences que les personnes migrantes ont eu du système de soins suisse sont susceptibles de peser sur la valeur qu'elles accordent aux actions engagées en leur faveur. Il nous a semblé dès lors utile de chercher à connaître les difficultés que ces personnes avaient pu rencontrer à cette occasion. Pour la plupart des migrants, les aspects de la qualité de la communication médicale sont essentiels. Une part non négligeable d'entre eux fait état de propos vécus comme dénigrants au sein même de ces consultations. Plusieurs personnes interviewées rapportent des épisodes relevant d'un racisme rampant alors même qu'on y reconnaît des propos que certains soignants en situation délicate ont pu tenir de manière défensive. Le témoignage le plus explicite à ce sujet est celui de cette femme qui dénonce le racisme de sa gynécologue lors d'une discussion sur les rapports sexuels après ablation de l'utérus pour un cancer:

[...] II y a des gens antipathiques, des racistes qui vous disent des choses : "Qu'est-ce que vous faites là ? »... Ou bien une doctoresse qui m'a dit après l'opération... si j'ai encore des rapports avec mon mari. Je lui ai dit, "j'ai de la peine et tout, heureusement que mon mari est âgé, il comprend, mais on a d'autres façons de nous aimer ». Et la doctoresse qui me sort, "ah bon, vous avez épousé un vieux en attendant sa mort comme un cadeau qui vous tombe du ciel ». Ça m'a beaucoup touchée... 
L'identification projective est une défense qui surgit inopinément chez des soignants choqués dans leurs valeurs et en difficulté pour préserver leur jugement de l'autre. Ceci n'est évidemment pas une justification ni n'ôte le vécu d'agression. Il reste cependant possible d'ouvrir le débat. D'une part avec les soignants qui doivent s'interroger sur la diversité des représentations de la maladie et sur la sensibilité des personnes malades, a fortiori en situation de migration. D'autre part, avec les malades qui peuvent tolérer et discuter la différence de comportements si, dans les pays d'accueil, figure explicitement un discours sur la variété des réactions entre peuples. 